REVIEW ARTICLE

https://doi.org/10.1038/s41467-019-09716-7

\title{
Humanising the mouse genome piece by piece
}

\author{
Fei Zhu ${ }^{1,3}$, Remya R. Nair ${ }^{2,3}$, Elizabeth M.C. Fisher (D) $^{1} \&$ \\ Thomas J. Cunningham (1D ${ }^{2}$
}

To better understand human health and disease, researchers create a wide variety of mouse models that carry human DNA. With recent advances in genome engineering, the targeted replacement of mouse genomic regions with orthologous human sequences has become increasingly viable, ranging from finely tuned humanisation of individual nucleotides and amino acids to the incorporation of many megabases of human DNA. Here, we examine emerging technologies for targeted genomic humanisation, we review the spectrum of existing genomically humanised mouse models and the insights such models have provided, and consider the lessons learned for designing such models in the future.

\footnotetext{
$\mathrm{G}$ enetically modified mice facilitate the analysis of gene function and regulation, as well as the dissection of disease mechanisms. Transgenic mice carrying human complementary DNAs (cDNAs) or mini-genes at non-endogenous loci have been around for decades (herein called 'classic transgenics') ${ }^{1}$, as have gene-targeted mice in which changes are made to specific DNA sequences in the mouse genome. Researchers increasingly combine these approaches and turn to the targeted genomic humanisation of mice, creating 'knock-in' mouse models in which a mouse sequence is replaced by the human orthologous $\mathrm{DNA}^{2}$. Compared with the thousands of classic transgenic mouse strains that mostly carry human DNA as randomly inserted, multicopy transgenes, the number of targeted genomically humanised mouse strains is low (Tables 1 and 2). Such mice are designed to have greater physiological relevance than their classic transgenic counterparts, which includes maintaining the correct genomic context of a gene of interest to preserve physiological expression levels and correct spatiotemporal expression patterns. Where non-coding human sequences are incorporated, humanspecific regulation and human gene splice isoforms may be maintained. In addition, the translated protein is expected to display the unique biochemical properties of the human gene, including potentially unique deleterious properties when mutated.

Genomically humanised mouse strains with physiological levels of gene expression tend to have slower, milder phenotypes than transgenic overexpression models. However, these genetargeted animals avoid overexpression artefacts, ectopic expression, and mutations resulting from random integration ${ }^{3,4}$ that can arise in transgenic models. Endogenous expression levels are particularly important when modelling dosage-sensitive genes, such as the RNA binding proteins C9orf $72^{5}$, TARDBP (Tar DNA-binding protein that encodes TDP-43) ${ }^{6,7}$ or FUS (fused in sarcoma) $)^{8,9}$. Mutations in these genes can be causative for the neurodegenerative disease amyotrophic lateral sclerosis (ALS), which is characterised by progressive motor neuron loss and paralysis. In contrast, overexpression of the human wild-type FUS alone is sufficient to cause motor neuron degeneration and other deficits in transgenic mice, thereby limiting the study of
}

\footnotetext{
${ }^{1}$ Department of Neuromuscular Diseases, Institute of Neurology, University College London, London WC1N 3BG, UK. ${ }^{2}$ Mammalian Genetics Unit, MRC Harwell Institute, Oxfordshire OX11 ORD, UK. ${ }^{3}$ These authors contributed equally: Fei Zhu, Remya R. Nair. Correspondence and requests for materials should be addressed to E.M.C.F. (email: elizabeth.fisher@ucl.ac.uk)
} 


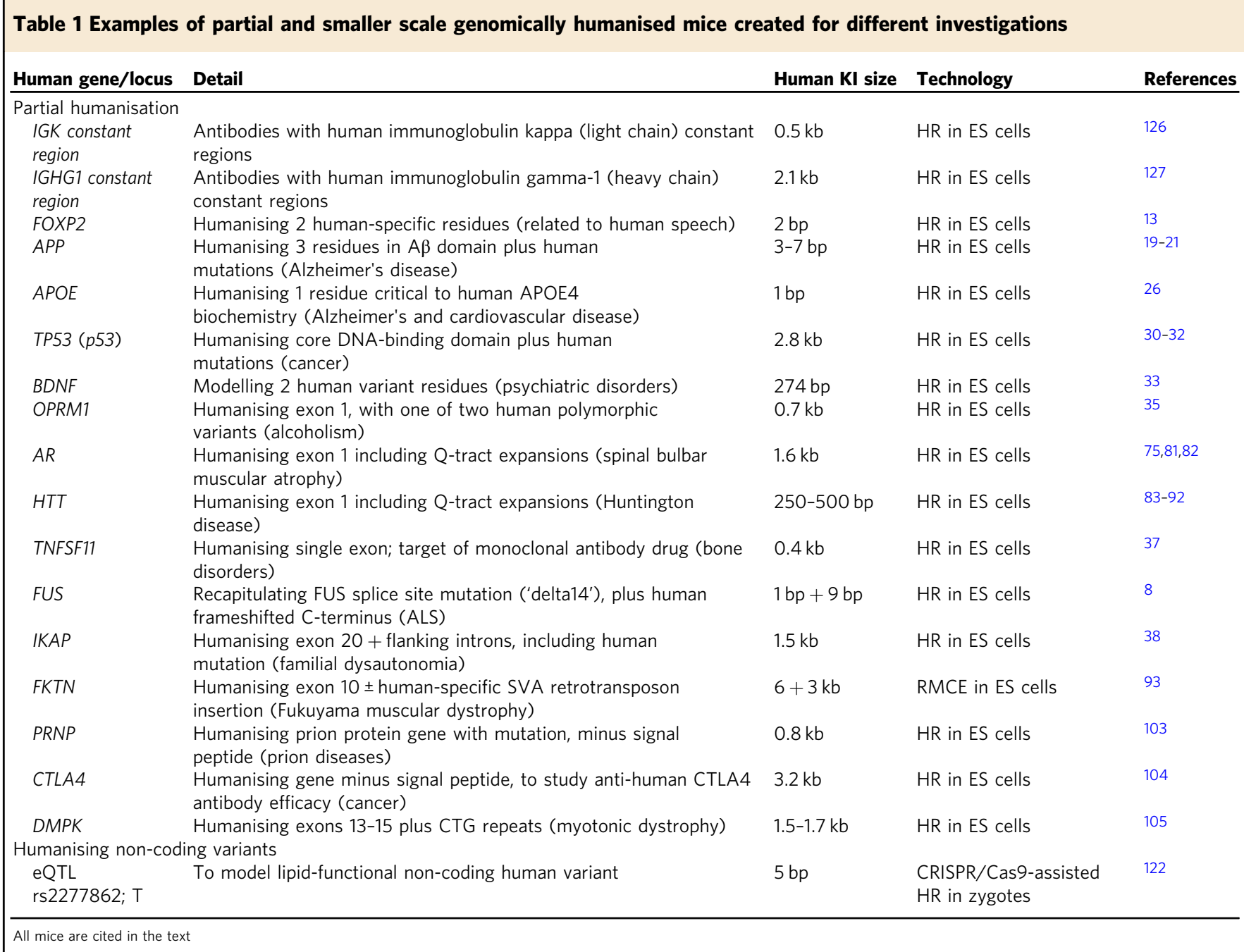

pathogenic mutations ${ }^{10}$ and making it necessary to tightly control gene dosage in these animal models.

Thus, genomically humanised mice offer refined models of human biology and pathology, as well as models for testing small molecule drug therapies, antibody therapeutics ${ }^{11}$, and gene therapies such as antisense oligonucleotides ${ }^{12}$, as we will discuss below.

As well as gaining biological insight, by analysing existing genomically humanised mice researchers are also gathering data for future genomic humanisation design and strategy-to guide decisions with potentially far reaching consequences on phenotype. Here, we first consider the biological, pathomechanistic and translational insights that have been gained from genomically humanised mice, and then we look at lessons learned so far which may influence future humanisation strategies. New technologies and resources (Box 1 and Fig. 1) are changing researchers' abilities to genomically humanise the mouse, but they will only be successful if they are efficient, reliable, and reproducible and without significant off-target effects.

\section{Insights from humanised mouse models}

Consider the question asked. It is possible to humanise individual base pairs and thus codons within a mouse gene to determine which amino acids are critical for the function or pathology under investigation. Experimenters can also choose a technically more challenging strategy of humanising entire exons, genes or even chromosomes, should the problem at hand not be addressable by smaller genomic alterations. Now that creating genomically humanised mice is becoming more straight forward (although still not routine), the questions these models are meant to address need to be clear a priori.

Humanisation of specific amino acids. Individual codon(s) for a protein of interest can be humanised to determine whether the encoded amino acid has a critical impact on function. An early example comes from an investigation of the evolution of human communication: Foxp $2^{\text {hum }}$ mice express a FOXP2 protein that has two 'human' amino acids that normally are not present in mouse FOXP2 (substitutions T302N and N324S). These residues are thought to have been positively selected during human evolution, possibly because of enhancing effects on speech and language ${ }^{13}$. Foxp $2^{\text {hum }}$ mice have an intriguing gain-of-function phenotype, showing altered behavioural learning dynamics and increased corticostriatal synaptogenesis, which is opposite to the phenotype observed in Foxp2 null mice. Thus, these finely humanised animals are helping to shed light on evolutionary adaptations of the human brain potentially required for the acquisition of speech capabilities ${ }^{14,15}$.

Another example of humanising specific codons to recreate human protein chemistry and to study the effects of mutation comes from research on Alzheimer's disease (AD). In humans, the APP (amyloid precursor protein) gene, when mutated or present in three copies, can cause early-onset $\mathrm{AD}$, which involves the deposition of the central $\mathrm{A} \beta$ peptide portion of the APP 
Table 2 Examples of whole gene and larger-scale genomically humanised mice created for different investigations

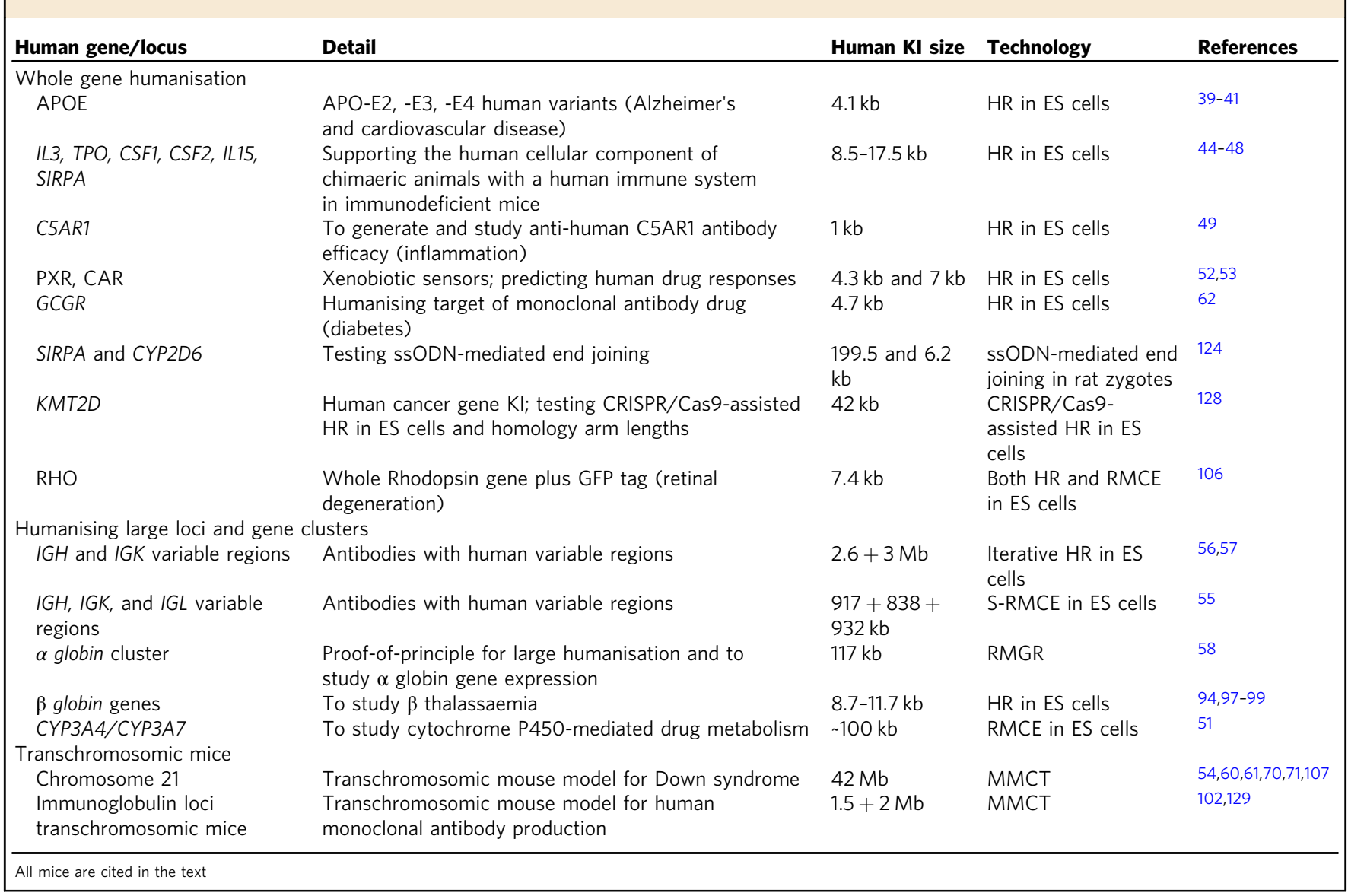

\section{Box 1 Technology old and new}

Classical DNA targeting using plasmid vectors and homologous recombination (HR) in mouse embryonic stem (ES) cells has been the predominant technology to date to create genomically humanised knock-in mice (Fig. 1a), applied successfully to humanise loci < 15 kb (Tables 1 and 2), but no larger because of constraints on the insert size of plasmids used for targeting vectors. Among the earliest results were mice created to produce partially humanised antibodies by targeted humanisation of the mouse immunoglobulin kappa (light chain) and gamma-1 (heavy chain) constant regions ${ }^{126,127}$. With the development of new vectors that carry tens of $\mathrm{kb}$ or more of insert DNA, such as bacterial artificial chromosomes (BACs), yeast artificial chromosome (YACs), human artificial chromosome (HACs) and mammalian artificial chromosomes (MACs), introducing much larger regions of human sequence became possible. BAC vectors combined with ES cell technologies have been used to humanise whole genes, gene clusters and even megabase-sized loci, via large-scale HR (Fig. 1a). Alternatively, recombinase enzymes and their short site-specific recombination sequences have been exploited for 'recombinase-mediated cassette exchange' (RMCE; Fig. 1b) and 'recombinase-mediated genomic exchange' (RMGE) strategies ${ }^{55-58 .}$ While these methods have worked well, new CRISPR-based technologies for knocking-in exogenous DNA are changing the research landscape, albeit with potential off-target effects ${ }^{130}$. For example, CRISPR/Cas9-assisted HR in ES cells improves targeting efficiency, reducing the time and resources required to screen ES cell clones. This method was used to assess the optimal length of homology arms required for HR and to create a new strain of mice in which the Kmtd2 (lysine (K)-specific methyltransferase 2D) gene was replaced by its $\sim 42 \mathrm{~kb}$ genomic human orthologue (KMTD2), from the methionine start codon to the stop codon ${ }^{128}$.

Targeting with CRISPR/Cas9 is developing rapidly, and is increasing our capacity for genomic humanisation of rodent genomes. CRISPR/Cas9-assisted $\mathrm{HR}$ in zygotes, using a single-stranded oligodeoxynucleotide (sSODN) as a repair template (also known as 'easiCRISPR'), is sufficient for efficient editing in zygotes without selection and therefore without genomic scarring. This has great potential for introducing pathogenic and other changes into large humanised regions, or for humanising small genomic regions: the current size limit for ssODN donors is $\sim 2 \mathrm{~kb}$, although this boundary is constantly challenged ${ }^{131,132}$ (Fig. 1c).

CRISPR/Cas9 combined with sSODN-mediated end joining is a striking new method for knocking-in long sequences directly to zygotes without the need to clone homology arms. Instead, a pair of short ssODNs harbouring short regions of homology, split between the donor template and target site, is used (Fig. 1d). This method was applied to knock-in $200 \mathrm{~kb}$ of human DNA including the SIRPA (signal-regulatory protein alpha) locus from a BAC vector, while removing the rat Sirpa homologous region, and in a separate experiment, to replace $58 \mathrm{~kb}$ of the rat Cyp2d (cytochrome P450, 2d) cluster with a $6.2 \mathrm{~kb}$ human CYP2D6 gene ${ }^{124}$.

Finally, the mouse genome can also be genomically humanised by adding entire human chromosomes, with the purpose of modelling the complexities of human aneuploidy disorders, such as Down syndrome, which arises from trisomy of chromosome 21. This can be achieved by microcell-mediated chromosome transfer, whereby chromosomes in a human cell line are tagged with an antibiotic selectable marker; the cells are subsequently forced to multinucleate, which facilitates the isolation of individual chromosomes and the creation of 'microcells'. These are fused with mouse ES cells, and used to generate mice via chimaera formation in blastocysts $54,60,61,102,129$. 
a Homologous recombination (HR) in ES cells

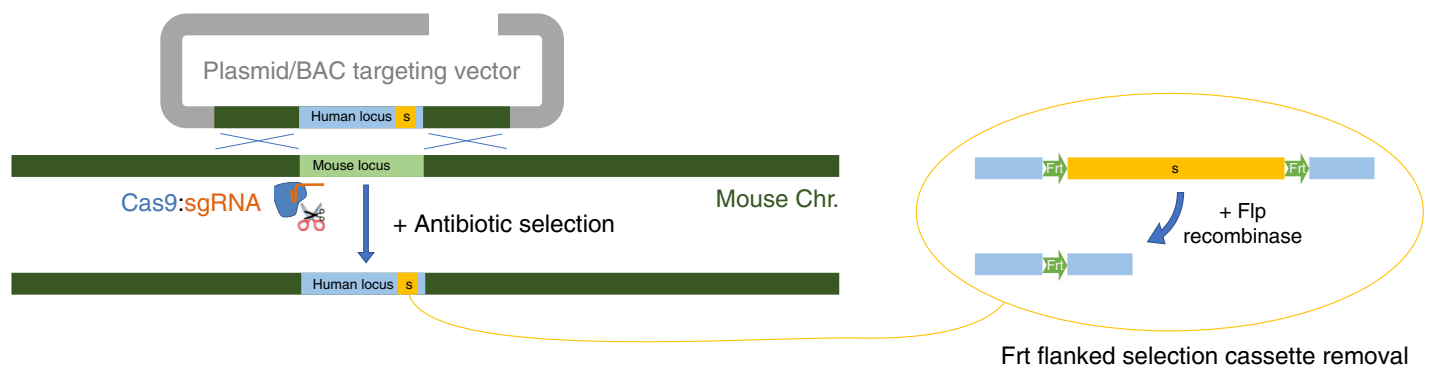

$\mathbf{b}_{\text {Recombinase mediated cassette exchange in ES cells }}$

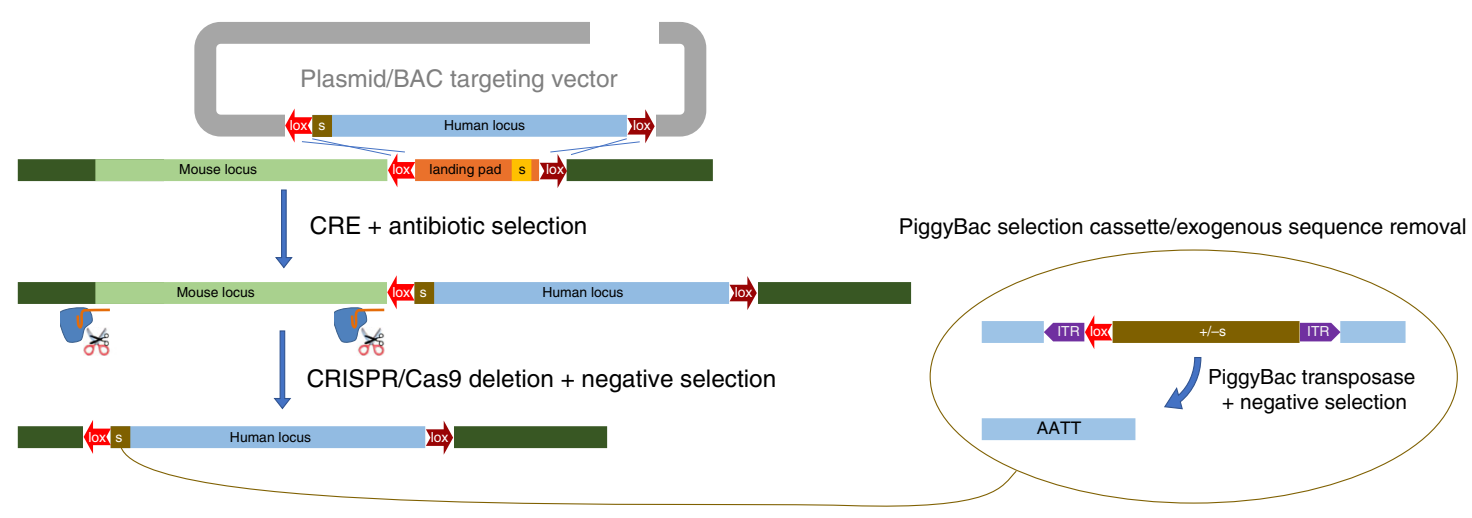

C CRISPR/Cas9 assisted HR using short or long ssODN donors in zygotes
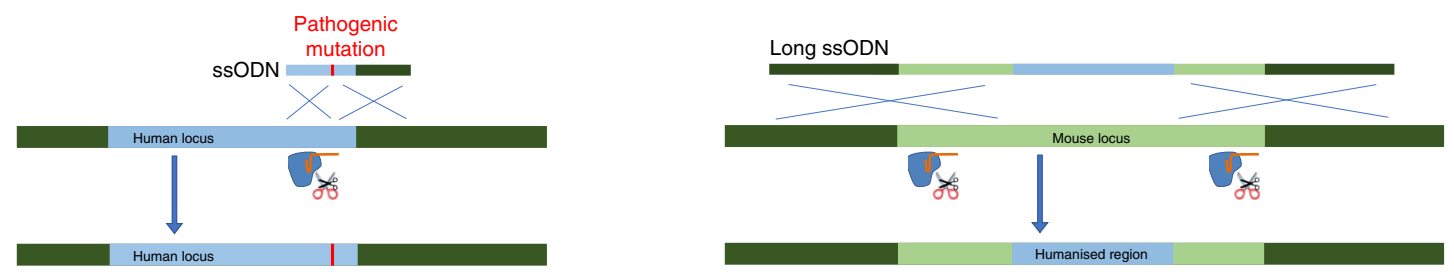

d CRISPR/Cas9 assisted HR via ssODN mediated end-joining in zygotes

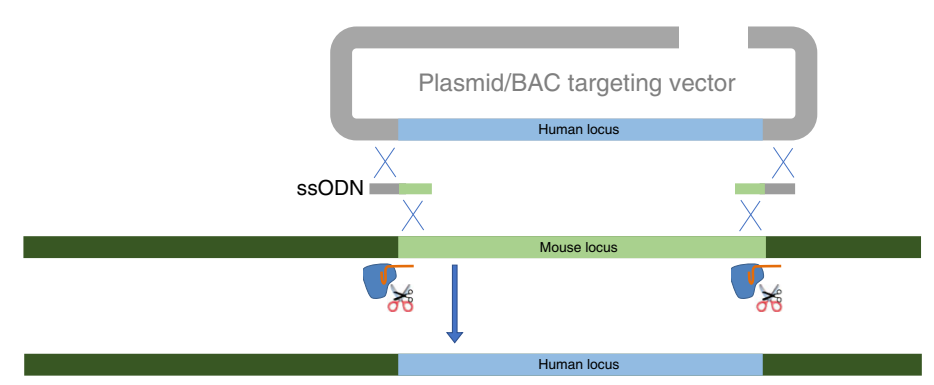

protein as extracellular plaques in the brain ${ }^{16,17}$. However, mouse APP does not form the typical amyloid plaques found in human $\mathrm{AD}$. The reason appears to be that full-length mouse and human APP proteins differ by 17 amino acids, notably at three key residues within the central $A \beta$ region that make the human protein more 'aggregatable' than that of the mouse ${ }^{18}$. AD researchers first humanised this $A \beta$ domain in 1996 by targeting the three critical $A \beta$ region codons, plus adding in a 'Swedish' familial $\mathrm{AD}$ mutation (causing two amino-acid substitutions immediately adjacent to the $\beta$-secretase site in APP), which resulted in enhanced $A \beta$ production ${ }^{19}$. This came at a time when creating humanised knock-in animals was unusual and only 5 years after the first human $A P P$ mutations had been described in early-onset familial $\mathrm{AD}^{16}$. Subsequently, other $\mathrm{AD}$ researchers followed this same targeted humanisation approach for $A P P$, but adding in other well-defined key familial AD mutations that affect $\mathrm{A} \beta$ processing within the rest of the protein ${ }^{19-21}$. This has resulted in extremely successful models of amyloid deposition and some of these mice are currently among the most widely used models in $\mathrm{AD}$ research, taken up by hundreds of labs worldwide $^{21}$. Recently, the widely used 'NL-F' mice (carrying both the 'Swedish' (NL) and 'Iberian' (F) APP mutations) have been instrumental in showing that $A \beta$ plaques can be seeded from cadaveric human pituitary growth hormone extracts into mice, 
Fig. 1 Targeted genomic humanisation technologies. a HR in ES cells has been used to humanise loci up to $200 \mathrm{~kb}$ (and beyond, using iterative targeting). A plasmid, or BAC, targeting vector carrying human sequence flanked by homology arms is transfected into ES cells by electroporation. Addition of Cas9: sgRNA, generating a targeted double strand break, increases HR efficiency. An antibiotic resistance selectable marker is included to enrich for ES cells harbouring the desired recombination. Selection cassettes are commonly flanked by frt sites for later excision by FLP recombinase, leaving a single frt genomic scar. b Recombinase-mediated cassette exchange (RMCE) can be used to humanise up to $200 \mathrm{~kb}$ loci (can also be employed iteratively). In this example, a landing pad is first inserted at the target locus via HR (see part $\mathbf{a}$ ), consisting of a selection cassette flanked by heterotypic lox sites. The same lox sites are inserted either side of the orthologous human locus within a BAC vector, which when electroporated into landing pad-harbouring ES cells will recombine in the presence of CRE recombinase. Cas9:sgRNA pairs can subsequently be utilised to delete the mouse locus. As an alternative to FLP/frt recombination, selection cassettes and other exogenous sequences can be flanked by PiggyBac inverted terminal repeats (ITR), which when inserted at an AATT recognition site, leave no genomic scar once excised with PiggyBac transposase. PiggyBAC transposition is less efficient than FLP/frt recombination, thus positive-negative selection cassettes $(+/-s$ ) such as HPRT (in HPRT-/- ES) or puro $\Delta$ TK are used. c Introducing pathogenic mutations into humanised alleles can be achieved by HR in zygotes using a ssODN ( $150 \mathrm{bp}$ ) donor template combined with a locus-specific Cas9:sgRNA (no selection required). A similar strategy can be used for small-scale humanisation projects (small genes or partial humanisation) using a long ssODN ( $<2 \mathrm{~kb})$ as a donor template and a pair of Cas9:sgRNAs. $\mathbf{d}$ Knock-in of large inserts (up to $200 \mathrm{~kb}$ ) in both mouse and rat zygotes has been achieved by combining Cas9:sgRNAs and short ssODN donors with hybrid homology at the break-points between donor and target site to facilitate HR

and thus potentially also humans, highlighting the need to carefully assess the risks for $\mathrm{A} \beta$ seed contamination via medical procedures such as dura mater grafting 22 .

Fine-scale genomic humanisation of mouse genes can also be used to address the question of how different alleles of a protein of interest may play different roles in phenotypes. This is essential for understanding the biochemical interactions relevant to variation in human disease. To give an example, Apolipoprotein $\mathrm{E}$ (APOE) is a protein involved in lipoprotein binding and metabolism. Humans have three $A P O E$ alleles (-E2, -E3 and -E4) that differ at residues 112 and 158. The APOE4 variant, which is associated with susceptibility to cardiovascular disease and $\mathrm{AD}$ risk, uniquely harbours Arg112 that causes another residue Arg61 to be exposed in the N-terminal domain enabling Arg61 to interact with the C- terminal domain (-E2 and -E3 variants neither have an exposed Arg61 nor such a domain interaction). However, while the single mouse APOE allele has APOE4-like Arg112, it lacks the human-specific Arg61 residue, lacks the domain interaction, and instead preferentially binds to high density lipoproteins, akin to the properties of human APOE3. When mouse APOE was humanised solely at residue 61 (Thr61 to Arg61) its lipoprotein binding preference changed to very low density lipoproteins (VLDLs) (akin to human APOE4), resulting in cognitive deficits and increased susceptibility to cardiovascular disease, consistent with human APOE4-dependent clinical features. In cardiovascular disease, increased binding of APOE4 to VLDLs leads to LDL receptor downregulation and increased LDL plasma levels (i.e., 'bad cholesterol' that can build up in the arteries $)^{23}$; such an increase in LDL cholesterol is also a risk factor for dementia, which is thought to alter the deposition and/or clearance of $A \beta$, although the mechanism is not fully understood $^{24}$. These key findings highlight that a specific domain interaction could be an important therapeutic target for ameliorating impairments in APOE4 in humans ${ }^{25-27}$.

Humanising individual domains or exons. While introducing individual human amino acids can be critical for modelling human biology and pathology in mouse, this is not always sufficient. A dominant human pathogenic amino-acid mutation in the $V C P$ (valosine containing protein) gene results in inclusion body myopathy associated with Paget disease of bone and frontotemporal dementia (IBMPFD) and ALS, but the same heterozygous mutation has little effect in mice ${ }^{28}$. For many models, the humanisation of specific stretches of amino acids or whole protein domains is key to answering mechanistic questions. One example that illustrates this comes from cancer research. The core DNA-binding domain of p53 (TP53, transformation related protein 53) is a hotspot for human cancer-associated mutations, and, thus, of outstanding interest for drilling into mechanisms of carcinogenesis. However, carcinogen-induced mutations in mice do not affect the same residues as in humans ${ }^{29}$. Therefore, to create mouse models suitable for understanding cellular processes in human cancer, key exons 4-9 of mouse $p 53$ (Trp53), which include the core DNA-binding domain, were humanised, resulting in the translation of a chimeric mouse:human $\mathrm{p} 53$ protein ${ }^{30}$. Subsequent introduction of human cancer-causing mutations gave new insights into how p53 mutations cause disruption to DNA damage-sensing and -response pathways ${ }^{31}$; and shed light on a human polymorphic p53 variant that may be associated with increased cancer risk in individuals of African descent ${ }^{32}$.

To study the effects of allelic variation on brain-derived neurotrophic factor (BDNF) function, a region of $274 \mathrm{bp}$ of the human gene was inserted into the mouse $B d n f$ gene, including one of two allelic variants: Val66 or Met66 $6^{33}$. Val66 is the major allele and also present in mouse, whereas Met66 in humans reduces BDNF activity-dependent secretion, leads to structural changes in the brain, and has been associated with psychiatric disorders and stress. Comparison of these mice showed that BDNF modulates competition in axonal branching ${ }^{33}$, which may be involved in Met66 allele-associated neurological dysfunction. Humanisation in this case, while providing a useful model to study human variation, was not critical since others have created a BDNF Met66 knock-in allele without humanisation to study anxiety-related phenotypes to good effect ${ }^{34}$.

Two allelic variants of the OPRM1 gene have also been modelled by humanisation of the first coding exon of mouse Oprm1, including one of two variants: the major 118A allele and the minor $118 \mathrm{G}$ allele linked to alcoholism. The whole first exon was humanised because of the evolutionary divergence between mouse and human in this region, although the requirement for this extent of humanisation is untested. These humanised lines showed that the $118 \mathrm{G}$ allele enhances ventral striatal dopamine responses to alcohol, while also helping to refine translational studies by supporting the hypothesis that the $118 \mathrm{G}$ allele confers an enhanced response to the opiate antagonist drug naltrexone; a disputed link previously inferred from clinical studies ${ }^{35,36}$.

Continuing with the theme of preclinical translational research, monoclonal antibodies are an essential tool of modern medicine, but human and mouse epitopes often differ, which can be problematic for in vivo testing of human monoclonal antibody therapies in mouse. Humanisation of a mouse epitope can overcome this problem. For example, a single exon of the Rankl (Tnfsf11, tumour necrosis factor ligand superfamily, member 11) gene was humanised to recapitulate the region of human RANKL 
targeted by the monoclonal antibody drug Denosumab, which inhibits osteoclastogenesis and increases bone density; the resulting mice were important for preclinical testing of the efficacy of the drug (non-humanised mice do not respond) in treating specific bone disorders in humans ${ }^{37}$.

Partial humanisation can also be useful to recreate humanspecific nonsense peptides that result from frameshift mutations that give rise to premature stop codons. For example, a spliceacceptor site mutation in the human FUS (Fused in sarcoma) gene that causes ALS leads to skipping of exon 14 and a frameshift in the coding sequence of exon 15 (resulting in a 14 amino-acid frameshifted C-terminus), followed by a premature stop codon. To recapitulate this mutation in the mouse, exon 15 of mouse Fus was humanised and the exon 14-skipping splice-acceptor site variant was introduced. Humanising only the splice site mutation would have resulted in a different and significantly longer frameshifted amino-acid sequence because of the differences in exon 15 between human and mouse. The partially humanised 'FUS-Delta14' mouse shows progressive motor neuron degeneration in a dominant, toxic gain-of-function manner and is currently one of the few physiological ALS models available. Importantly, the 14 amino-acid human-specific C-terminal frameshifted peptide has been instrumental in developing an antibody, which can be used to track the diseasecausing protein in both mouse and human FUS-Delta14expressing cells to help understand motor neuron death in ALS .

Partial genomic humanisation of mice also includes the introduction of critical disease-causing non-coding DNA sequences such as introns and regulatory elements. In a mouse model of the recessive disorder familial dysautonomia, the humanisation of a disease-causing intronic sequence resulted in skipping of exon 20 of the IKAP transcript (IKB kinase complexassociated protein) in the central nervous system, resulting in a premature stop codon and protein depletion, which is also observed in patients. While protein depletion in the mouse was not sufficient to recapitulate neurological phenotypes of the disease, this model has been used for proof-of-principle studies for developing new therapies aimed at restoring functional protein levels ${ }^{38}$.

Thus, the humanisation of individual domains, regions and introns to create chimeric transcripts and proteins has provided the scientific community with highly informative mouse models for understanding basic mechanisms and test systems for translational research.

Fully humanised genes and networks within the mouse context. The abundance of data from transgenic mice expressing human cDNAs, or genomic sequences from plasmid or BAC vectors, reassures that generally mouse and human proteins carry out equivalent functions, and that fundamental processes such as the recognition of splice boundaries are conserved between the two species. The relatively few examples of full genomic humanisation of mouse genes-i.e., the replacement of the entire protein coding region, including introns, with the human sequence-generally confirm that the human genes can successfully replace their mouse orthologues, albeit with sometimes minor changes in gene expression.

Fully genomically humanised mice have been created to recapitulate human biology and to achieve the greatest physiological relevance in disease modelling or therapeutic development, rather than to address questions of normal protein function. For example, the mouse Apoe gene discussed above has been fully genomically humanised in order to gain further insight into the role of APOE in disease, including the effects of the individual alleles. All three human $A P O E$ polymorphic variants (APOE2, $-E 3$ and $-E 4)$ were knocked-in to the mouse locus (all three coding exons plus intervening introns), placing them under the control of the mouse promoter. These models have been widely used to better understand the molecular basis for atherosclerosis and $\mathrm{AD}$ while also providing a means to test therapeutics in a humanised context ${ }^{39-42}$. We note that such models may be crossed in classical genetics approaches to provide insights into human biochemical pathways. Humanised Apoe mice, for example, have been crossed with Tau transgenic mice, which revealed that APOE4 aggravates tauopathy independent of amyloid- $\beta$ pathology 43 .

Under certain circumstances, it may be necessary to humanise multiple genes within a pathway or network to better model human biology. Chimeric mice harbouring a human immune system can be generated by engrafting immunodeficient mice with human haematopoietic stem cells; however, mouse cytokines poorly cross react with human receptors and are inadequate to support the maturation and function of these human immune cells. Cytokines can be administered to the mice to overcome this problem, but to achieve physiological expression levels and distribution, several cytokine genes have now been fully genomically humanised in mouse, and successfully support the human cellular component of chimeric animals. Used in combination, these knocked-in cytokine genes help improve the development and function of human monocytes, macrophages, and NK cells in the mouse environment ${ }^{44-48}$.

Moving onto translational research, a novel method for production of high affinity monoclonal antibodies against human Complement C5a Receptor 1 (C5AR1), a therapeutic target for various inflammatory conditions, was enabled by humanising the complete mouse C5ar1 gene ${ }^{49}$, using neutrophils from C5AR1 humanised mice (expressing high levels of human C5AR1) to immunise wild-type mice. C5AR1 humanised mice were subsequently successfully used for preclinical testing of the antibodies. For pharmacokinetics analysis, a number of xenobiotic sensors and drug metabolising enzymes have been fully humanised to help predict human drug responses ${ }^{50-53}$

Although there are relatively few published examples in which entire mouse genes have been replaced with the human gene, these genomically humanised animals are valid models for understanding human biology and pathology, and have provided tools and given insight that would not have been possible with studying the mouse orthologues alone.

Genome humanisation for investigating complex disease loci. The largest region of humanisation within the mouse genome extends over tens of megabases of DNA within an almost complete human chromosome 21 (Hsa21) ${ }^{54}$. In mice developed to produce human antibodies, humanised regions are several megabases in length ${ }^{55-57}$. In one of the first large-scale genomic humanisation studies of gene expression, the humanised region was almost $200 \mathrm{~kb}$ long, located in the $\alpha$ globin gene cluster ${ }^{58}$. In all these cases, perhaps surprisingly, phenotypes ranged from relatively mild to apparently no different from wild-type mice (other than for the production of human proteins). The largest published region of humanisation currently comes from studying the complex human chromosomal disorder Down syndrome, which is an aneuploidy syndrome resulting from trisomy of Hsa21. Modelling this disorder in mice poses a problem because the content of Hsa21 is distributed over three syntenic regions on mouse chromosomes 10,16 and $17^{59}$. Thus, transchromosomic mice ('Tc1' and others) were created via microcell-mediated chromosome transfer of Hsa21 into mouse embryonic stem (ES) cells and subsequent production of chimeric mice via blastocyst injection, followed by breeding of chimeras for germline 
transmission of the human chromosome $e^{54,60,61}$. Tc1 mice, which carry an extra $>42 \mathrm{Mb}$ of human DNA, recapitulate many features of Down syndrome, most of which are fairly mild (on the genetic backgrounds studied) and include neurological, cardiac and mandible phenotypes ${ }^{54}$. However, Tc1 mice lose the human chromosome stochastically, more from rapidly dividing cells such as those in the thymus, than, say those in the brain (where $\sim 67 \%$ of cells retain the human chromosome), and the resulting mosaicism probably contributes to the mildness of the phenotypes $^{54}$.

Efficient production of human monoclonal antibodies from human immunoglobulin IgG light and heavy chain knock-in mice has been achieved, by two different groups independently, by megabase-sized knock-in of the variable regions of both heavy and light chain human IgG loci ${ }^{55-57}$. Both groups implemented iterative targeting of kilobase-sized segments in ES cells using BAC vectors; one group used a homologous recombination (HR) strategy, while the other utilised recombinase-mediated cassette exchange (RMCE) alongside the PiggyBac transposase system for megabase-sized scarless editing. Remarkably, in both approaches, these knock-in mice show no functional impairments in their immune systems, which is in contrast to previous non-targeted humanisation efforts. They have been used for testing therapies against HIV-1 (human immunodeficiency virus-1) and developing human therapeutic antibodies to treat diabetes ${ }^{55-57,62,63}$. More recently, an alternative one-step approach was devised to knock-in pre-arranged human heavy chain sequences $(\sim 1.9 \mathrm{~kb}$; directly into zygotes) for generation of antibodies against critical HIV-1 epitopes ${ }^{64}$.

Genomic humanisation of large complex loci has shed light on important non-coding regulatory sequences, which would have been difficult in conventional mouse models. Humanisation of the $\alpha$ globin gene cluster, via recombinase-mediated genomic exchange (RMGE), was undertaken to study gene expression from this clinically important region and represented a proof-ofprinciple that humanising large genomic loci is possible. One outcome of this research was the finding that globin gene expression levels differ between the humanised mice and wildtype animals. Further dissection of the underlying cause showed that the HS-40 element in this cluster was critical for $\alpha$ globin gene expression within the human, but not the mouse locus ${ }^{58,65}$.

The surprising conclusion from reports of mice in which large regions of the genome have been replaced or human sequences added, is that phenotypes are generally mild without an apparent effect on mouse welfare. This is at least true for wild-type loci. However, even in the largest genomic humanisation effort reported to date, the Tc1 mouse model of Down syndrome, $<1 \%$ of the genome is human DNA.

\section{Lessons learned so far for humanisation strategies}

The rules of the genome are not fully understood, nor are the full phenotypic consequences of creating mice with human genomic DNA. However, although genomically humanised mice are relatively few, it is clear that they can give insight into biology and pathology that cannot necessarily be gained from conventional mouse models. Advances in genome engineering technologies, including CRISPR (clustered regularly interspaced short palindromic repeats (CRISPR)-Cas approaches (Box 1), mean that making humanised models is easier and faster than ever beforealthough still not a conventional route-such that even when the specifics of mouse:human transcript or protein differences are unknown, humanisation should be considered. However, the design of a humanisation strategy is far from routine, and a key question remains: how far to humanise (summarised in Fig. 2)?

To keep things simple or go full scale?. The decision whether to humanise just a codon, just a domain, just the coding region, or to go full scale and include introns and flanking regulatory regions depends on the questions being asked, as we have discussed above. Most genomically humanised mice in the literature have fine-scale changes within the coding sequence that result in chimeric human:mouse proteins, facilitating the study of critical aspects of protein biochemistry, without greatly altering mouse genome architecture.

To work with models that contain full-length human genes, perhaps in trying to produce human-specific splice patterns and regulation from intronic sequences, human genomic architecture

a

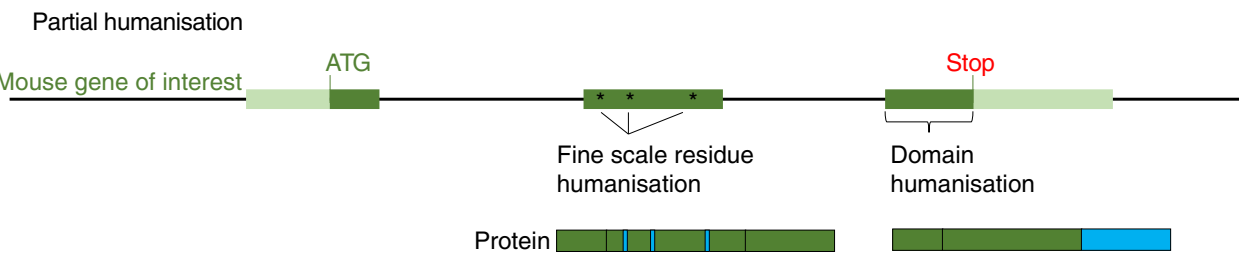

b

Full humanisation

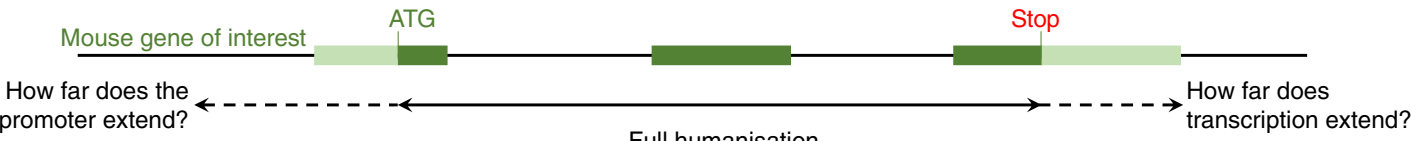

Full humanisation

Protein

Fig. 2 How far to humanise. A summary of considerations when deciding on the extent of targeted genomic humanisation for a given gene of interest. Dark green boxes represent exons, lines between exons are introns, light green boxes are UTRs and blue regions represent humanisation. a Partial humanisation typically involves humanising specific residues and/or domains of interest. Fine-scale humanisation of specific amino-acid residues can be performed in isolation if they have known biochemical differences from mouse to human, or if only a small number of residues need to be altered to achieve a human protein sequence. Specific domains or exons can be humanised if, for example, they are known to be critical for human disease. Examples of translated protein products are given. b Full humanisation involves humanising the whole gene, including introns, to attain translation of the full human protein, potentially including human-specific splicing patterns, and for maximum translational potential. 5' and 3'-UTRs, promoters, and other regulatory sequences can be included, on a case-by-case basis, if understanding of gene regulation is the question at hand, if gene clusters are to be humanised or if pathogenic mutations fall within such flanking regions 
has to be introduced. Mice have on average of $\sim 2.4$ splice isoforms per gene, whereas humans produce $\sim 3.4$ isoforms per gene $^{66}$ and individual splice isoforms can be disease causative. For example, the isoform ratios of TAU, encoded by MAPT, are critical in causing frontotemporal dementia, and while a genomically humanised MAPT mouse has not yet been described, encouragingly a human BAC transgenic recapitulates human MAPT splicing in mouse ${ }^{67}$. Thus, if the full repertoire of human splice isoforms is required, then full-scale humanisation across the gene of interest could be attempted.

Exclusion of introns, which might help to keep things simple in a humanisation strategy, can potentially be disruptive. For example, when the xenobiotic sensor PXR (Pregnane X receptor) was originally humanised, some introns were excluded-it was later discovered that this caused a mis-splicing event as a result of exons 7 and 8 fusion, leading to creation of a new splice-acceptor site and variant whereby exon 5 spliced to exon 8 . The gene was subsequently fully humanised to eliminate this variant ${ }^{52,53}$. Thus, and perhaps not surprisingly, in at least some cases, maintaining the human genome architecture is likely critical for normal gene expression in mouse.

We note that in moving towards translational research, the presence of the full-length human transcripts and proteins maximises the potential to test therapeutics relevant for use in humans. An excellent example of this comes from the need to determine the optimal dose of human antisense oligomer (ASO) therapies against the human rather than mouse mRNA sequences, because in the clinic the oligomer must bind to the human sequence; as, for example, in ASOs that modulate APOE levels ${ }^{42}$.

Considering flanking regulatory sequences. If an investigation warrants the genomic humanisation of an entire mouse gene, which is coding regions and internal introns, the decision over how far to extend into the $5^{\prime}$ and $3^{\prime}$ regions currently relies largely on a combination of limited information, expediency, and guesswork. It is essential to look closely at how far the gene of interest extends upstream and downstream, as apart from different transcripts, the gene may in addition produce alternative $5^{\prime}$ and $3^{\prime}$ exons that contain different untranslated regions (UTRs), which may affect gene regulation. However, even with this information, generally too little is known about the regulatory sequences within most genes to judge whether to humanise only from the first ATG to the last stop codon or to humanise from the start of the first 5' UTR (if known) to the end of the last $3^{\prime}$ UTR (if known) of alternative transcripts. For single genes, a conservative approach is to humanise between outermost known start and stop codons only. A riskier approach-but one that may result in more faithfully recapitulated human gene regulation-is to include the human UTRs. However, currently there is insufficient information to reliably predict the outcome of this strategy and to make generalisable rules.

A critically important feature for gene regulation is the gene promotor, but currently no good spatial definition of the promotors of most genes is available. Often the best that researchers can do is to assume that the CG-rich region upstream of a gene includes the promotor, and to look carefully for transcription factor binding sites within or nearby this genomic sequence. In fact, so far targeted humanisation of most single genes has not included promoter sequences, just in case critical promoter-enhancer interactions were to be disrupted. Furthermore, some regulatory elements may control genes long distances away ${ }^{68}$, for example, in human and mouse the $1 \mathrm{Mb}$-distal ZRS enhancer for Sonic hedgehog (Shh) is critical to limb-specific Shh expression $^{69}$.
One potential limitation of genomic humanisation strategies that include the promotor regions is that even though human and mouse transcription factors are conserved, orthologues do not necessarily have identical amino-acid sequences or recognise identical motifs. Therefore, mouse proteins might not correctly regulate the transcription of human genes. This problem was addressed to some extent by the Tcl mouse model of Down syndrome that carries Hsa21, mentioned above. The researchers investigating transcription factor binding found that mouse transcription factors did indeed bind to the human cognate sites, despite the sequences often being slightly different from the mouse orthologous sites. This result may allay some fears about introducing human promoters for human genes into the mouse cellular environment ${ }^{70}$. This result was in part corroborated by a recent RNA deep sequencing study of Hsa21 in both the Tc1 mouse and in human cells; ${ }^{71}$ specifically, mouse trans acting factors (such as transcription factors) appeared responsible for regulating humanised gene expression. This was evident since humanised gene expression levels in mouse closely correlated with levels of the mouse orthologous gene, and not the human gene in human cells ${ }^{71}$, in contrast to findings of the earlier study that suggested human expression levels were maintained ${ }^{70}$. Importantly for humanisation studies, splicing patterns of genes on the humanised chromosome in Tc1 mice closely recapitulated splicing patterns in human cells, even in human non-coding genes that do not have mouse orthologues ${ }^{71}$, indicating that regulation of splicing is intrinsically sequence dependent and that splicing machinery is well conserved between mouse and human.

Finally, expediency comes into play in targeting strategies when humanising a sequence might disrupt important mouse features. In a project we have underway, we find that the $3^{\prime}$-UTR of a mouse gene of interest overlaps the $5^{\prime}$-UTR of the adjacent gene, whereas in the human genome the two orthologues are separated by several $\mathrm{kb}$. In this case, our humanisation strategy extends only to the stop codon of the gene of interest, and no further, to ensure that the mouse 3'-UTR is not disrupted, and we do not inadvertently alter the regulation of the downstream gene.

Genomic humanisation of sequences that do not exist in mouse. Approximately 1\% of human genes do not have a mouse orthologue and vice versa ${ }^{72}$, and some of the $99 \%$ of homologous protein coding genes may exist in different copy numbers in the two species. For example, mice have a single Smn gene encoding the survival motor neuron protein, but humans have $S M N 1$ and up to four copies of its paralogue SMN2. SMN1 and SMN2 are almost identical but a single-nucleotide change in exon 7 of SMN2 results in $\sim 80 \%$ of its transcripts producing an unstable, truncated protein and only $\sim 20 \%$ of SMN2 mRNA encoding functional protein. This becomes critically important when studying type 1 spinal muscular atrophy (SMA), a recessive disease with an incidence of $\sim 1$ in 10,000 depending on the population, which arises from mutation in SMN1. Left untreated, children with SMA die before 4 years of age from respiratory failure due to lower motor neuron death ${ }^{73}$. This form of SMA has been modelled by strategies that include targeting human SMN2 into Smn null mice, for example, to recapitulate the human condition (ref. ${ }^{74}$ and papers therein).

We wish to raise for consideration the following (so far hypothetical, but possible) scenario: where human paralogs have taken on different functions, but only one mouse homologue exists, humanising the mouse gene may potentially only produce a protein that functions as a single human paralog, leaving the remaining functions of the other human paralog(s) absent from the mouse.

Triplet (and larger) repeat expansion diseases have not been found in mice, and yet are a well-known cause of neuromuscular, 
neurodegenerative and other disorders in humans. Genomic humanisation is a route to model these disorders in mice; for example, in humans, a CAG expansion in exon 1 of the androgen receptor $(A R)$ gene results in a polyglutamine (polyQ) tract in the protein and can lead to spinal bulbar muscular atrophy (SBMA). Mouse $A r$ exon 1 has significant sequence divergence from the human orthologue and so a model was created that humanised $1340 \mathrm{bp}$ of exon 1, including a human-specific 113residue polyQ-tract. This has proven successful in uncovering disease mechanisms and developing therapeutics for SBMA ${ }^{75-82}$.

Huntington disease (HD) is another polyQ-expansion neurodegenerative disease, which has been modelled extensively via $\mathrm{BAC}$ transgenesis (producing fast, neurodegenerative phenotypes) and via knock-in strategies (slowly progressive phenotypes). Humanised knock-in HD models consist of humanisation of exon 1 of the mouse Huntington $(\mathrm{Htt})$ gene, which includes a pathogenic polyQ-tract adjacent to a polypurine tract ${ }^{83-87}$ (both of these features show poor conservation between mouse and human). Such mice are available as an allelic series derived from natural expansion of the CAG repeat in vivo, ranging from those with mild molecular changes to those with progressive behavioural and motor dysfunction. Non-humanised knock-in HD mice (expanding the mouse polyQ tract alone) have also been generated with similar phenotypes; nevertheless, humanised models have been widely used to gain insights into disease pathophysiology, including characterising the timing of early neuropathological changes, identifying posttranslational modifications crucial to mutant $H T T$ pathology, and omics profiling to understand repeat length-dependent dysfunctions ${ }^{88-91}$. Humanisation has also allowed successful therapeutic testing of gene editing strategies targeting human exon 1 with the aim of excising the repeat expansion ${ }^{92}$.

When the genomic context of a disease-causing mutation is entirely specific to humans, humanisation is the only option. For example, an SVA retrovirus insertion into the $3^{\prime}$-UTR (located in exon 10) of the human FKTN (FUKUTIN) gene causes Fukuyama muscular dystrophy ${ }^{93}$. SVA retrotransposons are hominid specific and do not exist in mouse. Therefore, the whole of mouse Fktn exon 10 was humanised, with and without the SVA insertion, to replicate any sequence-specific effects the insertion may have on the host gene. Indeed, this turned out to be important as the SVA insertion caused an abnormal 3' splicing pattern in both the mouse model and human patients-leading to severely reduced protein expression. The humanised model mimics some-but not all-of the human disorder phenotype, and has been useful for understanding disease mechanism. This example demonstrates that introducing a wider genomic context to the disease-causing mutation can be essential for replicating pathogenic mechanisms ${ }^{93}$.

In genetically complex disorders, differences between mouse and human might be so prominent-both in genomic context and biology - that a considerable investment in understanding the locus or loci of interest has to be made before a detailed approach to modelling can be developed. $\beta$-Thalassaemia in humans results from mutations in the $\beta$ globin locus that lead to a reduction of functional adult $\beta$-globin protein levels. Simple replacement of the two mouse genes for adult $\beta$ globin with the single human $\beta$ globin gene containing an intronic causative $\beta$-thalassaemia $\beta$ IVS-2-654 mutation led to classic signs of $\beta$-thalassaemia intermedia in heterozygous mice ${ }^{94}$. These mice have been used to test therapies that either block (using spliceswitching oligonucleotides) or correct (via gene editing) the cryptic splice site introduced by the mutation ${ }^{95,96}$. However, divergence in sequence (four genes in mouse, five in human) and timing of embryonic-to-foetal-to-adult $\beta$-globin expression between mouse and human complicates modelling of both $\beta$-thalassaemia major and sickle $\beta$-thalassaemia-severe, recessive forms of anaemia characterised by a lack of functional adult $\beta$-globin. Human patients survive untreated for some time postnatally, supported by foetal $\beta$-globins, which do not fully switch to adult $\beta$-globins until 1 year of age. Simply knocking-out mouse adult $\beta$-globins fails to model these recessive anaemias because mice lack a bona fide foetal haemoglobin and the knockout mice are non-viable because mouse foetal liver erythropoiesis depends on adult $\beta$-globins. Thus, to model human recessive thalassaemias, a bespoke humanised approach was required that involved the replacement of the mouse adult $\beta$ globin genes with a delayed foetal-to-adult haemoglobin-switching transgene enabling survival to birth ${ }^{97,98}$. Postnatal survival was later further improved by introducing a hereditary persistence of foetal haemoglobin' mutation within the human $\gamma$-globin gene component ${ }^{99,100}$. Thus, gaining a detailed understanding of the architectural and expression differences between mouse and human loci may be necessary to inform how best to introduce human sequences into a mouse genome to obtain the most informative results.

Humanising entire chromosomes. The genome contains features beyond the primary genetic sequence that may affect humanisation strategies. A possible example of this arises in the Tc1 mouse model of Down syndrome, which stochastically loses Hsa21 over time, resulting in mosaic mice ${ }^{54}$. This may be due to the centrosomes of the mouse cells not properly recognising or interacting with the centromere of the human chromosome. New types of human artificial chromosomes (HACs), including those with mouse centromeric sequences, are likely to give greater stability to human chromosome-sized regions in mouse $\mathrm{s}^{101}$ Similar to the Tc1 model, microcell-mediated chromosome transfer was employed to create mice harbouring human minichromosomes carrying the entire immunoglobulin heavy and kappa light chain loci, combined with knock-out of the orthologous mouse loci, to successfully express fully human antibodies $^{102}$. Of note in this case, minichromosomes were found to be mitotically and meiotically unstable and mice had impaired Bcell development and partial immune deficiencies.

Full genomic humanisation is not always the best strategy. Finally, it may be beneficial not to humanise specific domains when they, for example, have important functions in determining cellular localisation. In humanisation of both Prnp (Prion protein) and Ctla4 (cytotoxic T lymphocyte-associated 4), the mouse signal peptide sequence in each of these genes was retained, to avoid potential problems with translocation to the cell membrane; since signal peptides are cleaved, this should have no effect of the biochemistry of the mature protein ${ }^{103,104}$.

Unexpected effects. The phenotype of a genetic mouse modeltransgenic or genomically humanised-is not always predictable, but when unexpected outcomes arise, such models can provide valuable insight from studying the mechanism of why the observed outcome is different from expectation. As an example, to model myotonic dystrophy, the $3^{\prime}$ end of the Dmpk (dystrophia myotonica protein kinase) gene was humanised, including the addition of 84 CTG repeats in the $3^{\prime}$-UTR, which leads to pathology in humans. In Dmpk-humanised mice, however, this repeat number failed to produce a pathogenic phenotype. Nevertheless, this model gave important insight into the somatic instability of the repeats, and provided evidence that repeat stability is associated with the activity of mismatch-repair (MMR) machinery ${ }^{105}$. 
Similarly, in larger-scale humanising of the introns and exons of a gene, the mouse environment (chromosomal or cellular) may cause unexpected phenotypes. Currently, this is unpredictable and must be assessed on a case-by-case basis, but does not preclude working with such animals. For example, the large-scale humanisation of the a globin locus (knocking-in $117 \mathrm{~kb}$ of human DNA, deleting $85 \mathrm{~kb}$ of syntenic mouse DNA) resulted in significantly reduced expression $(40 \%$ of mouse a globin expression levels) of a globin from the humanised locus, but the developmental pattern of a globin expression was retained and histological markers were normal, allowing evaluation of the mechanistic outcomes of point and indel mutations at this locus $^{58}$.

Genome architecture can be disrupted by scars in the genomic landscape (i.e., introduction of exogenous sequences that could, for example, disrupt promoters or enhancers and affect gene regulation). An experiment to humanise rhodopsin included fusion of green fluorescent protein (GFP) at the C-terminal end, in order to visualise Rhodopsin-expressing cells in the retina with high sensitivity; however, the GFP fusion generated a recessive allele, unexpectedly causing death of rod photoreceptor cells, thereby providing a useful, even though unintended, model for retinal neurodegeneration ${ }^{106}$. In the same study, other humanised alleles were generated that also included the presence of recombinase recognition lox sites flanking Rhodopsin-GFP, which significantly reduced translation of the gene, and further exacerbated retinal degeneration. Thus, introduction of exogenous sequences into a locus should be carefully considered and evaluated for unintended effects. Certain forms of genomic scarring, such as frt sites left behind from exogenously inserted selection cassettes (needed during genome engineering in ES cells; see Fig. 1a), can now readily be avoided by using the PiggyBac transposase system or CRISPR/Cas9 targeting in zygotes (which does not require selection, and therefore leaves no genomic scar) (Fig. 1b, c).

Another unexpected, but informative, outcome of humanisation comes from the Tc1 mouse model, which was produced to understand Down syndrome phenotypes, but in fact also gave insight into the expression of primate-specific repeats. Over $50 \%$ of the human genome is derived from repetitive elements, mostly from transposable elements that can act as regulatory DNA and whose activity is controlled by several different mechanisms. Placing Hsa21 into the mouse nuclear environment resulted in transcriptional activation of transposon-derived human regulatory regions, which could potentially influence gene expression of nearby transcripts on this chromosome; among other results, this highlighted the latent regulatory potential of human-specific elements ${ }^{107}$.

Importantly, we also wish to highlight the need to work with a wild-type genomically humanised animal as a control, where possible, when studying genomically humanised mutants. This is to ensure that unexpected phenotypes that derive from the human DNA per se are distinguished from those associated with the mutation, in particular when introducing many $\mathrm{kb}$ - or $\mathrm{Mb}$-sized stretches of DNA.

When far is too far. Just as important as the technical and strategic considerations regarding genomic humanisation, are the ethical issues-how far can humanising the mouse genome go while still having a mouse? This is an unresolved question that was discussed in a report by the UK Academy of Medical Sciences ('Animals containing human material', 2011), which at the time cautiously concluded there were no new ethical challenges. However, since then the field of genome editing has exploded with new technologies and these considerations may have to be revisited in the near future. This issue is of course also pertinent to the mouse:human cellular chimeras that have been made for many years with the purpose of studying the immune system and which are now also being created, for example, to better understand brain function and dysfunction ${ }^{108,109}$.

\section{Genomic humanisation going forward}

The technology for targeted genomic humanisation of the mouse requires further development to streamline approaches and to simplify working with long and challenging sequences such as expanded repeats. In that regard, CRISPR/Cas systems are changing rapidly, offering great potential for genomic humanisation that is only just beginning to be realised. Modified Cas9 variants, including base editors and variants with alternative protospacer adjacent motif specificity, now permit more precise editing than ever before ${ }^{110,111}$, whereas structural or copy number variants can be modelled in mice following humanisation of any given gene $\mathrm{s}^{112}$.

Nevertheless, many technical challenges remain. For example, several important human disease genes are now known to undergo repeat-associated non-ATG (RAN) translation-often producing peptides in all three or even all six reading frames (when taking into account translation in the reverse direction $)^{113}$. Producing humanised mouse models that carry stable repeats to understand associated pathomechanisms is not yet routine. Furthermore, for at least some of these repeats the flanking sequence contexts may be important for disease manifestation $^{114}$, meaning that, in addition, the entire human gene sequence has to be knocked-in as a control.

Even in cases of relatively straight forward humanisation of a single wild-type gene, as described in the examples above, it is not always clear how far one should extend genomic humanisation in order to most faithfully recapitulate human gene expression in a mouse context. In eukaryotes, for example, it largely remains to be determined where and how transcription terminates, which may be many bp or even $\mathrm{kb}$ beyond the polyadenylation signal in a transcript. Transcription terminator proteins are involved in releasing mRNAs and RNA Polymerase II from each other ${ }^{115,116}$, and this is coupled to cleavage and polyadenylation of the $3^{\prime}$ end of mRNAs and associated with the effects of transcription boundary-associated RNAs, which include terminus-associated RNAs ${ }^{117}$. Termination can occur at different sites $3^{\prime}$ of the polyadenylation signal, and usually it is not known how far to humanise beyond the $3^{\prime}$-UTR of a gene. Yet, these sequences can have profound effects on transcription and translation ${ }^{118}$.

Similarly, codon usage is clearly important for translation, affecting, for example, mRNA stability, ribosome binding, mRNA processivity, and other phenomena ${ }^{119}$. However, as mouse and human are only separated by 75 million years of evolution, it is assumed that codon usage will have similar effects in both species, but this may not be the case for specific genes.

Thus, the optimal strategy for genomic humanisation is likely to be only discernible on a gene-by-gene basis following considerable prior investment in gathering data on human gene expression in human cells and tissues. However, existing models clearly show the utility of working with current knowledge, and have in fact helped to find new regulatory sequences that would have been hard to find otherwise $2,58,65$.

In making genomically humanised mice, unexpected insights about coding regions can be gained; for example, for some pathogenic human alleles, such as the A53T mutation in SNCA that causes Parkinson's disease, the human disease variant is the wild-type sequence in the mouse. This well-known phenomenon occurs at least in some cases because of compensatory 
evolutionary changes at specific sites elsewhere in the gene/ protein ${ }^{120,121}$. Thus, humanisation of the complete gene, with the normal human sequence (aberrant in mouse) and the disease human allele (wild-type in mouse) may provide a route to studying phenotype. Alternatively, the evolutionary compensation of variant residues could occur within other genes that interact with SNCA, meaning that multiple genes might have to be humanised to yield a phenotype of interest.

In modelling human disease in an age of personalised medicine, humanisation of a single allele will not be sufficient to address the great variability between individuals, including response to treatment and clinical trajectories. This remains a challenge for mouse modelling in general, not just for creating genomically humanised models. Furthermore, humanisation of a single gene may not be sufficient to understand biology or pathology-particularly when protein complexes or ligand-receptor interactions are involved. Thus, at a minimum, the between-species conservation of these interactions should be considered. Future approaches to humanised mouse models may lie in humanising entire networks or pathways.

Genome wide association studies have identified thousands of disease risk loci in the human genome, the majority of which lie in non-coding regions. Modelling non-coding variants in animals is challenging, due to the lack of evolutionary sequence conservation in many non-coding regions. Recently, liver-associated expression quantitative trait loci (eQTL) were identified via screening in hepatocyte cell lines derived from a cohort of healthy volunteers $^{122}$, as part of a study to identify lipid-functional genes and variants, and to better understand human complex traits. In the same study, one of the lead eQTLs (rs2277862; T) was modelled in mice via CRISPR-Cas9 mutagenesis in zygotes, including humanisation of four surrounding nucleotides such that the wider region became identical to the human sequence, leading to significant reduction in expression of the nearby CPNE1 gene, whose function is still to be determined. Thus, technological advances in genome engineering combined with the ever improving understanding of genomic architecture render genetic modelling of non-coding variants an exciting future prospect $^{123}$.

The development of CRISPR/Cas technologies means that the mouse is no longer exceptional and in fact data on genomically humanised rats and pigs are already emerging ${ }^{124,125}$. Future humanisation strategies may thus involve a range of model organisms, which inevitably means new ethical boundaries will have to be defined. Finally, to facilitate a reduction in the use of animals for research purposes, humanised mouse models can and should be studied in parallel with human tissue samples and human in vitro models, which will at the same time further help in obtaining a more complete picture of human biology and pathology.

Received: 20 September 2018 Accepted: 23 March 2019

Published online: 23 April 2019

\section{References}

1. Gordon, J. W. \& Ruddle, F. H. Integration and stable germ line transmission of genes injected into mouse pronuclei. Science 214, 1244-1246 (1981).

2. Devoy, A., Bunton-Stasyshyn, R. K., Tybulewicz, V. L., Smith, A. J. \& Fisher, E. M. Genomically humanized mice: technologies and promises. Nat. Rev. Genet. 13, 14-20 (2011).

3. Goodwin, L. O. et al. Large-scale discovery of mouse transgenic integration sites revleals frequent structural variation and insertional mutagenesis. Preprint at bioRxiv: https://doi.org/10.1101/236307 (2017).

4. Tosh, J. L. et al. The integration site of the APP transgene in the J20 mouse model of Alzheimer's disease. Wellcome Open Res. 2, 84 (2017).
5. Batra, R. \& Lee, C. W. Mouse models of C9orf72 hexanucleotide repeat expansion in amyotrophic lateral sclerosis/frontotemporal dementia. Front. Cell. Neurosci. 11, 196 (2017).

6. White, M. A. et al. TDP-43 gains function due to perturbed autoregulation in a Tardbp knock-in mouse model of ALS-FTD. Nat. Neurosci. 21, 552-563 (2018).

7. Fratta, P. et al. Mice with endogenous TDP-43 mutations exhibit gain of splicing function and characteristics of amyotrophic lateral sclerosis. EMBO J. https://doi.org/10.15252/embj.201798684 (2018).

8. Devoy, A. et al. Humanized mutant FUS drives progressive motor neuron degeneration without aggregation in 'FUSDelta14' knockin mice. Brain 140, 2797-2805 (2017)

9. Scekic-Zahirovic, J. et al. Toxic gain of function from mutant FUS protein is crucial to trigger cell autonomous motor neuron loss. EMBO J. 35, 1077-1097 (2016).

10. Mitchell, J. C. et al. Overexpression of human wild-type FUS causes progressive motor neuron degeneration in an age- and dose-dependent fashion. Acta Neuropathol. 125, 273-288 (2013).

11. Elgundi, Z., Reslan, M., Cruz, E., Sifniotis, V. \& Kayser, V. The state-of-play and future of antibody therapeutics. Adv. Drug Deliv. Rev. 122, 2-19 (2017).

12. Schoch, K. M. \& Miller, T. M. Antisense oligonucleotides: translation from mouse models to human neurodegenerative diseases. Neuron 94, 1056-1070 (2017).

13. Enard, W. et al. A humanized version of Foxp2 affects cortico-basal ganglia circuits in mice. Cell 137, 961-971 (2009).

14. Schreiweis, C. et al. Humanized Foxp2 accelerates learning by enhancing transitions from declarative to procedural performance. Proc. Natl. Acad. Sci. USA 111, 14253-14258 (2014).

15. Chen, Y. C. et al. Foxp 2 controls synaptic wiring of corticostriatal circuits and vocal communication by opposing Mef2c. Nat. Neurosci. 19, 1513-1522 (2016).

16. Goate, A. et al. Segregation of a missense mutation in the amyloid precursor protein gene with familial Alzheimer's disease. Nature 349, 704-706 (1991).

17. Wiseman, F. K. et al. A genetic cause of Alzheimer disease: mechanistic insights from Down syndrome. Nat. Rev. Neurosci. 16, 564-574 (2015).

18. Xu, G. et al. Murine Abeta over-production produces diffuse and compact Alzheimer-type amyloid deposits. Acta Neuropathol. Commun. 3, 72 (2015).

19. Reaume, A. G. et al. Enhanced amyloidogenic processing of the beta-amyloid precursor protein in gene-targeted mice bearing the Swedish familial Alzheimer's disease mutations and a "humanized" Abeta sequence. J. Biol. Chem. 271, 23380-23388 (1996).

20. Li, H. et al. Vascular and parenchymal amyloid pathology in an Alzheimer disease knock-in mouse model: interplay with cerebral blood flow. Mol. Neurodegener. 9, 28 (2014).

21. Saito, T. et al. Single App knock-in mouse models of Alzheimer's disease. Nat. Neurosci. 17, 661-663 (2014).

22. Purro, S. A. et al. Transmission of amyloid-beta protein pathology from cadaveric pituitary growth hormone. Nature 564, 415-419 (2018).

23. Mahley, R. W. Apolipoprotein E: from cardiovascular disease to neurodegenerative disorders. J. Mol. Med. (Berl) 94, 739-746 (2016).

24. Huynh, T. V., Davis, A. A., Ulrich, J. D. \& Holtzman, D. M. Apolipoprotein E and Alzheimer's disease: the influence of apolipoprotein $\mathrm{E}$ on amyloid-beta and other amyloidogenic proteins. J. Lipid Res. 58, 824-836 (2017).

25. Adeosun, S. O. et al. Cognitive deficits and disruption of neurogenesis in a mouse model of apolipoprotein E4 domain interaction. J. Biol. Chem. 289, 2946-2959 (2014).

26. Raffai, R. L., Dong, L. M., Farese, R. V. Jr. \& Weisgraber, K. H. Introduction of human apolipoprotein E4 "domain interaction" into mouse apolipoprotein E. Proc. Natl. Acad. Sci. USA 98, 11587-11591 (2001).

27. Eberle, D. et al. Apolipoprotein E4 domain interaction accelerates dietinduced atherosclerosis in hypomorphic Arg-61 apoe mice. Arterioscler. Thromb. Vasc. Biol. 32, 1116-1123 (2012).

28. Clemen, C. S. et al. The heterozygous R155C VCP mutation: toxic in humans! Harmless in mice? Biochem. Biophys. Res. Commun. 503, 2770-2777 (2018).

29. Dumaz, N. et al. The role of UV-B light in skin carcinogenesis through the analysis of p53 mutations in squamous cell carcinomas of hairless mice. Carcinogenesis 18, 897-904 (1997).

30. Luo, J. L. et al. Knock-in mice with a chimeric human/murine p53 gene develop normally and show wild-type p53 responses to DNA damaging agents: a new biomedical research tool. Oncogene 20, 320-328 (2001).

31. Song, H., Hollstein, M. \& Xu, Y. p53 gain-of-function cancer mutants induce genetic instability by inactivating ATM. Nat. Cell Biol. 9, 573-580 (2007).

32. Jennis, M. et al. An African-specific polymorphism in the TP53 gene impairs p53 tumor suppressor function in a mouse model. Genes Dev. 30, 918-930 (2016). 
33. Cao, L. et al. Genetic modulation of BDNF signaling affects the outcome of axonal competition in vivo. Curr. Biol. 17, 911-921 (2007).

34. Chen, Z. Y. et al. Genetic variant BDNF (Val66Met) polymorphism alters anxiety-related behavior. Science 314, 140-143 (2006).

35. Ramchandani, V. A. et al. A genetic determinant of the striatal dopamine response to alcohol in men. Mol. Psychiatry 16, 809-817 (2011).

36. Bilbao, A. et al. A pharmacogenetic determinant of mu-opioid receptor antagonist effects on alcohol reward and consumption: evidence from humanized mice. Biol. Psychiatry 77, 850-858 (2015).

37. Kostenuik, P. J. et al. Denosumab, a fully human monoclonal antibody to RANKL, inhibits bone resorption and increases BMD in knock-in mice that express chimeric (murine/human) RANKL. J. Bone Miner. Res. 24, 182-195 (2009).

38. Bochner, R. et al. Phosphatidylserine increases IKBKAP levels in a humanized knock-in IKBKAP mouse model. Hum. Mol. Genet. 22, 2785-2794 (2013).

39. Sullivan, P. M., Mezdour, H., Quarfordt, S. H. \& Maeda, N. Type III hyperlipoproteinemia and spontaneous atherosclerosis in mice resulting from gene replacement of mouse Apoe with human Apoe`2. J. Clin. Invest. 102, 130-135 (1998).

40. Sullivan, P. M. et al. Targeted replacement of the mouse apolipoprotein E gene with the common human APOE3 allele enhances diet-induced hypercholesterolemia and atherosclerosis. J. Biol. Chem. 272, 17972-17980 (1997).

41. Knouff, C. et al. Apo E structure determines VLDL clearance and atherosclerosis risk in mice. J. Clin. Invest. 103, 1579-1586 (1999).

42. Huynh, T. V. et al. Age-dependent effects of apoE reduction using antisense oligonucleotides in a model of beta-amyloidosis. Neuron 96, 1013-1023 (2017).

43. Shi, Y. et al. ApoE4 markedly exacerbates tau-mediated neurodegeneration in a mouse model of tauopathy. Nature 549, 523-527 (2017).

44. Herndler-Brandstetter, D. et al. Humanized mouse model supports development, function, and tissue residency of human natural killer cells. Proc. Natl. Acad. Sci. USA 114, E9626-E9634 (2017)

45. Rathinam, C. et al. Efficient differentiation and function of human macrophages in humanized CSF-1 mice. Blood 118, 3119-3128 (2011).

46. Rongvaux, A. et al. Development and function of human innate immune cells in a humanized mouse model. Nat. Biotechnol. 32, 364-372 (2014).

47. Rongvaux, A. et al. Human thrombopoietin knockin mice efficiently support human hematopoiesis in vivo. Proc. Natl. Acad. Sci. USA 108, 2378-2383 (2011).

48. Willinger, T. et al. Human IL-3/GM-CSF knock-in mice support human alveolar macrophage development and human immune responses in the lung. Proc. Natl. Acad. Sci. USA 108, 2390-2395 (2011).

49. Lee, H. et al. Human C5aR knock-in mice facilitate the production and assessment of anti-inflammatory monoclonal antibodies. Nat. Biotechnol. 24, 1279-1284 (2006).

50. Scheer, N., Snaith, M., Wolf, C. R. \& Seibler, J. Generation and utility of genetically humanized mouse models. Drug Discov. Today 18, 1200-1211 (2013).

51. Hasegawa, M. et al. Quantitative prediction of human pregnane X receptor and cytochrome P450 3A4 mediated drug-drug interaction in a novel multiple humanized mouse line. Mol. Pharmacol. 80, 518-528 (2011).

52. Scheer, N., Ross, J., Kapelyukh, Y., Rode, A. \& Wolf, C. R. In vivo responses of the human and murine pregnane $\mathrm{X}$ receptor to dexamethasone in mice. Drug Metab. Dispos. 38, 1046-1053 (2010).

53. Scheer, N. et al. A novel panel of mouse models to evaluate the role of human pregnane $\mathrm{X}$ receptor and constitutive androstane receptor in drug response. J. Clin. Invest. 118, 3228-3239 (2008).

54. O'Doherty, A. et al. An aneuploid mouse strain carrying human chromosome 21 with Down syndrome phenotypes. Science 309, 2033-2037 (2005).

55. Lee, E. C. et al. Complete humanization of the mouse immunoglobulin loci enables efficient therapeutic antibody discovery. Nat. Biotechnol. 32, 356-363 (2014).

56. Macdonald, L. E. et al. Precise and in situ genetic humanization of $6 \mathrm{Mb}$ of mouse immunoglobulin genes. Proc. Natl. Acad. Sci. USA 111, 5147-5152 (2014).

57. Murphy, A. J. et al. Mice with megabase humanization of their immunoglobulin genes generate antibodies as efficiently as normal mice. Proc. Natl. Acad. Sci. USA 111, 5153-5158 (2014).

58. Wallace, H. A. et al. Manipulating the mouse genome to engineer precise functional syntenic replacements with human sequence. Cell 128, 197-209 (2007).

59. Choong, X. Y., Tosh, J. L., Pulford, L. J. \& Fisher, E. M. Dissecting Alzheimer disease in Down syndrome using mouse models. Front. Behav. Neurosci. 9, 268 (2015)

60. Shinohara, T. et al. Mice containing a human chromosome 21 model behavioral impairment and cardiac anomalies of Down's syndrome. Hum. Mol. Genet. 10, 1163-1175 (2001).
61. Hernandez, D., Mee, P. J., Martin, J. E., Tybulewicz, V. L. J. \& Fisher, E. M. C. Transchromosomal mouse embryonic stem cell lines and chimeric mice that contain freely segregating segments of human chromosome 21. Hum. Mol. Genet. 8, 923-933 (1999).

62. Okamoto, H. et al. Glucagon receptor blockade with a human antibody normalizes blood glucose in diabetic mice and monkeys. Endocrinology 156, 2781-2794 (2015).

63. Sok, D. et al. Priming HIV-1 broadly neutralizing antibody precursors in human Ig loci transgenic mice. Science 353, 1557-1560 (2016)

64. Lin, Y. C. et al. One-step CRISPR/Cas9 method for the rapid generation of human antibody heavy chain knock-in mice. $E M B O \mathrm{~J}$. https://doi.org/ 10.15252/embj.201899243 (2018).

65. Vernimmen, D. et al. Chromosome looping at the human alpha-globin locus is mediated via the major upstream regulatory element (HS -40). Blood 114, 4253-4260 (2009).

66. Lee, Y. \& Rio, D. C. Mechanisms and regulation of alternative pre-mRNA splicing. Annu. Rev. Biochem. 84, 291-323 (2015).

67. Johnson, S. J. \& Wade-Martins, R. A BACwards glance at neurodegeneration: molecular insights into disease from LRRK2, SNCA and MAPT BACtransgenic mice. Biochem. Soc. Trans. 39, 862-867 (2011).

68. Sanyal, A., Lajoie, B. R., Jain, G. \& Dekker, J. The long-range interaction landscape of gene promoters. Nature 489, 109-113 (2012).

69. Lettice, L. A. et al. Disruption of a long-range cis-acting regulator for Shh causes preaxial polydactyly. Proc. Natl. Acad. Sci. USA 99, 7548-7553 (2002)

70. Wilson, M. D. et al. Species-specific transcription in mice carrying human chromosome 21. Science 322, 434-438 (2008).

71. Deveson, I. W. et al. Universal alternative splicing of noncoding exons. Cell Syst. 6, 245-255 (2018).

72. Waterston, R. H. et al. Initial sequencing and comparative analysis of the mouse genome. Nature 420, 520-562 (2002).

73. Verhaart, I. E. C. et al. Prevalence, incidence and carrier frequency of 5qlinked spinal muscular atrophy - a literature review. Orphanet J. Rare Dis. 12, 124 (2017).

74. Bebee, T. W., Dominguez, C. E. \& Chandler, D. S. Mouse models of SMA: tools for disease characterization and therapeutic development. Hum. Genet. 131, 1277-1293 (2012).

75. Albertelli, M. A., Scheller, A., Brogley, M. \& Robins, D. M. Replacing the mouse androgen receptor with human alleles demonstrates glutamine tract length-dependent effects on physiology and tumorigenesis in mice. Mol. Endocrinol. 20, 1248-1260 (2006).

76. Chua, J. P. et al. Transcriptional activation of TFEB/ZKSCAN3 target genes underlies enhanced autophagy in spinobulbar muscular atrophy. Hum. Mol. Genet. 23, 1376-1386 (2014).

77. Chua, J. P. et al. Disrupting SUMOylation enhances transcriptional function and ameliorates polyglutamine androgen receptor-mediated disease. J. Clin. Invest. 125, 831-845 (2015).

78. Giorgetti, E. et al. Rescue of metabolic alterations in AR113Q skeletal muscle by peripheral androgen receptor gene silencing. Cell. Rep. 17, 125-136 (2016).

79. Milioto, C. et al. Beta-agonist stimulation ameliorates the phenotype of spinal and bulbar muscular atrophy mice and patient-derived myotubes. Sci. Rep. 7, 41046 (2017).

80. Poort, J. E., Rheuben, M. B., Breedlove, S. M. \& Jordan, C. L. Neuromuscular junctions are pathological but not denervated in two mouse models of spinal bulbar muscular atrophy. Hum. Mol. Genet. 25, 3768-3783 (2016).

81. Yu, Z. et al. Androgen-dependent pathology demonstrates myopathic contribution to the Kennedy disease phenotype in a mouse knock-in model. J. Clin. Invest. 116, 2663-2672 (2006).

82. Yu, Z. et al. Abnormalities of germ cell maturation and sertoli cell cytoskeleton in androgen receptor 113 CAG knock-in mice reveal toxic effects of the mutant protein. Am. J. Pathol. 168, 195-204 (2006).

83. Menalled, L. B. et al. Comprehensive behavioral and molecular characterization of a new knock-in mouse model of Huntington's disease: zQ175. PLoS ONE 7, e49838 (2012).

84. Wheeler, V. C. et al. Long glutamine tracts cause nuclear localization of a novel form of huntingtin in medium spiny striatal neurons in HdhQ92 and HdhQ111 knock-in mice. Hum. Mol. Genet. 9, 503-513 (2000).

85. Menalled, L. B., Sison, J. D., Dragatsis, I., Zeitlin, S. \& Chesselet, M. F. Time course of early motor and neuropathological anomalies in a knock-in mouse model of Huntington's disease with 140 CAG repeats. J. Comp. Neurol. 465, $11-26$ (2003)

86. White, J. K. et al. Huntingtin is required for neurogenesis and is not impaired by the Huntington's disease CAG expansion. Nat. Genet. 17, 404-410 (1997).

87. Levine, M. S. et al. Enhanced sensitivity to $\mathrm{N}$-methyl-D-aspartate receptor activation in transgenic and knockin mouse models of Huntington's disease. $J$. Neurosci. Res. 58, 515-532 (1999).

88. Cariulo, C. et al. Phosphorylation of huntingtin at residue T3 is decreased in Huntington's disease and modulates mutant huntingtin protein conformation. Proc. Natl. Acad. Sci. USA 114, E10809-E10818 (2017). 
89. Menalled, L. B. et al. Early motor dysfunction and striosomal distribution of Huntingtin microaggregates in Huntington's disease knock-in mice. J. Neurosci. 22, 8266-8276 (2002).

90. Langfelder, P. et al. Integrated genomics and proteomics define huntingtin CAG length-dependent networks in mice. Nat. Neurosci. 19, 623-633 (2016).

91. Smith, G. A. et al. Progressive axonal transport and synaptic protein changes correlate with behavioral and neuropathological abnormalities in the heterozygous Q175 KI mouse model of Huntington's disease. Hum. Mol. Genet. 23, 4510-4527 (2014).

92. Yang, S. et al. CRISPR/Cas9-mediated gene editing ameliorates neurotoxicity in mouse model of Huntington's disease. J. Clin. Invest. 127, 2719-2724 (2017).

93. Taniguchi-Ikeda, M. et al. Pathogenic exon-trapping by SVA retrotransposon and rescue in Fukuyama muscular dystrophy. Nature 478, 127-131 (2011).

94. Lewis, J. et al. A common human beta globin splicing mutation modeled in mice. Blood 91, 2152-2156 (1998).

95. Svasti, S. et al. RNA repair restores hemoglobin expression in IVS2-654 thalassemic mice. Proc. Natl. Acad. Sci. USA 106, 1205-1210 (2009).

96. Bahal, R. et al. In vivo correction of anaemia in beta-thalassemic mice by gammaPNA-mediated gene editing with nanoparticle delivery. Nat. Commun. 7, 13304 (2016).

97. Huo, Y. et al. Humanized mouse model of Cooley's anemia. J. Biol. Chem. 284, 4889-4896 (2009).

98. Wu, L. C. et al. Correction of sickle cell disease by homologous recombination in embryonic stem cells. Blood 108, 1183-1188 (2006).

99. Huo, Y., McConnell, S. C. \& Ryan, T. M. Preclinical transfusion-dependent humanized mouse model of beta thalassemia major. Blood 113, 4763-4770 (2009).

100. Huo, Y. et al. Humanized mouse models of Cooley's anemia: correct fetal-toadult hemoglobin switching, disease onset, and disease pathology. Ann. NY Acad. Sci. 1202, 45-51 (2010).

101. Uno, N., Abe, S., Oshimura, M. \& Kazuki, Y. Combinations of chromosome transfer and genome editing for the development of cell/animal models of human disease and humanized animal models. J. Hum. Genet. 63, 145-156 (2018).

102. Tomizuka, K. et al. Double trans-chromosomic mice: maintenance of two individual human chromosome fragments containing Ig heavy and kappa loci and expression of fully human antibodies. Proc. Natl. Acad. Sci. USA 97, 722-727 (2000).

103. Kitamoto, T. et al. Humanized prion protein knock-in by Cre-induced sitespecific recombination in the mouse. Biochem. Biophys. Res. Commun. 222, 742-747 (1996).

104. Lute, K. D. et al. Human CTLA4 knock-in mice unravel the quantitative link between tumor immunity and autoimmunity induced by anti-CTLA-4 antibodies. Blood 106, 3127-3133 (2005).

105. van den Broek, W. J. A. A. Somatic expansion behaviour of the (CTG)n repeat in myotonic dystrophy knock-in mice is differentially affected by Msh3 and Msh6 mismatch-repair proteins. Hum. Mol. Genet. 11, 191-198 (2002).

106. Chan, F., Bradley, A., Wensel, T. G. \& Wilson, J. H. Knock-in human rhodopsin-GFP fusions as mouse models for human disease and targets for gene therapy. Proc. Natl. Acad. Sci. USA 101, 9109-9114 (2004).

107. Ward, M. C. et al. Latent regulatory potential of human-specific repetitive elements. Mol. Cell 49, 262-272 (2013).

108. Espuny-Camacho, I. et al. Hallmarks of Alzheimer's disease in stem-cellderived human neurons transplanted into mouse brain. Neuron 93, 1066-1081 (2017).

109. Real, R. et al. In vivo modeling of human neuron dynamics and Down syndrome. Science https://doi.org/10.1126/science.aau1810 (2018).

110. $\mathrm{Hu}, \mathrm{J}$. H. et al. Evolved Cas9 variants with broad PAM compatibility and high DNA specificity. Nature 556, 57-63 (2018).

111. Komor, A. C., Kim, Y. B., Packer, M. S., Zuris, J. A. \& Liu, D. R. Programmable editing of a target base in genomic DNA without doublestranded DNA cleavage. Nature 533, 420-424 (2016).

112. Birling, M. C. et al. Efficient and rapid generation of large genomic variants in rats and mice using CRISMERE. Sci. Rep. 7, 43331 (2017).

113. Cleary, J. D. \& Ranum, L. P. New developments in RAN translation: insights from multiple diseases. Curr. Opin. Genet. Dev. 44, 125-134 (2017).

114. Tabet, R. et al. CUG initiation and frameshifting enable production of dipeptide repeat proteins from ALS/FTD C9ORF72 transcripts. Nat. Commun. 9, 152 (2018).

115. Loya, T. J. \& Reines, D. Recent advances in understanding transcription termination by RNA polymerase II. F1000Res. https://doi.org/10.12688/ f1000research.8455.1 (2016).

116. Porrua, O., Boudvillain, M. \& Libri, D. Transcription termination: variations on common themes. Trends Genet. 32, 508-522 (2016).

117. Yu, D., Ma, X., Zuo, Z., Wang, H. \& Meng, Y. Classification of transcription boundary-associated RNAs (TBARs) in animals and plants. Front. Genet. 9, 168 (2018)
118. Tushev, G. et al. Alternative 3' UTRs modify the localization, regulatory potential, stability, and plasticity of mRNAs in neuronal compartments. Neuron 98, 495-511 (2018).

119. Hanson, G. \& Coller, J. Codon optimality, bias and usage in translation and mRNA decay. Nat. Rev. Mol. Cell Biol. 19, 20-30 (2018).

120. Gao, L. \& Zhang, J. Why are some human disease-associated mutations fixed in mice? Trends Genet. 19, 678-681 (2003).

121. Jordan, D. M. et al. Identification of cis-suppression of human disease mutations by comparative genomics. Nature 524, 225-229 (2015).

122. Pashos, E. E. et al. Large, diverse population cohorts of hiPSCs and derived hepatocyte-like cells reveal functional genetic variation at blood lipidassociated loci. Cell Stem. Cell 20, 558-570 (2017).

123. Laber, S. \& Cox, R. D. Mouse models of human GWAS hits for obesity and diabetes in the post genomic era: time for reevaluation. Front. Endocrinol. (Lausanne) 8, 11 (2017).

124. Yoshimi, K. et al. ssODN-mediated knock-in with CRISPR-Cas for large genomic regions in zygotes. Nat. Commun. 7, 10431 (2016).

125. Yan, S. et al. A Huntingtin knockin pig model recapitulates features of selective neurodegeneration in Huntington's disease. Cell 173, 989-1002 (2018).

126. Zou, Y. R., Gu, H. \& Rajewsky, K. Generation of a mouse strain that produces immunoglobulin-kappa chains with human constant regions. Science 262, 1271-1274 (1993).

127. Zou, Y. R., Muller, W., Gu, H. \& Rajewsky, K. Cre-loxP-mediated gene replacement: a mouse strain producing humanized antibodies. Curr. Biol. 4, 1099-1103 (1994).

128. Baker, O. et al. The contribution of homology arms to nuclease-assisted genome engineering. Nucleic Acids Res. 45, 8105-8115 (2017).

129. Tomizuka, K. et al. Functional expression and germline transmission of a human chromosome fragment in chimaeric mice. Nat. Genet. 16, 133-143 (1997).

130. Anderson, K. R. et al. CRISPR off-target analysis in genetically engineered rats and mice. Nat. Methods 15, 512-514 (2018).

131. Quadros, R. M. et al. Easi-CRISPR: a robust method for one-step generation of mice carrying conditional and insertion alleles using long ssDNA donors and CRISPR ribonucleoproteins. Genome. Biol. 18, 92 (2017).

132. Codner, G. F. et al. Application of long single-stranded DNA donors in genome editing: generation and validation of mouse mutants. BMC Biol. 16, 70 (2018).

\section{Acknowledgements}

We thank Peter Oliver and Lydia Teboul (MRC Harwell Institute) for critical reading of this manuscript and thoughtful improvements.

\section{Author contributions}

F.Z., R.R.N., E.M.C.F., T.J.C. researched the information for this paper; E.M.C.F. conceived the work; F.Z., R.R.N., E.M.C.F., T.J.C. drafted the manuscript and R.R.N, E.M.C.F, T.J.C. wrote the manuscript.

\section{Additional information}

Competing interests: The authors declare no competing interests.

Reprints and permission information is available online at http://npg.nature.com/ reprintsandpermissions/

Journal peer review information: Nature Communications thanks the anonymous reviewers for their contribution to the peer review of this work.

Publisher's note: Springer Nature remains neutral with regard to jurisdictional claims in published maps and institutional affiliations.

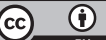

Open Access This article is licensed under a Creative Commons Attribution 4.0 International License, which permits use, sharing, adaptation, distribution and reproduction in any medium or format, as long as you give appropriate credit to the original author(s) and the source, provide a link to the Creative Commons license, and indicate if changes were made. The images or other third party material in this article are included in the article's Creative Commons license, unless indicated otherwise in a credit line to the material. If material is not included in the article's Creative Commons license and your intended use is not permitted by statutory regulation or exceeds the permitted use, you will need to obtain permission directly from the copyright holder. To view a copy of this license, visit http://creativecommons.org/ licenses/by/4.0/.

(C) The Author(s) 2019 